\title{
A Randomized Controlled Trial of an Additional Funding Intervention to Improve Clinical Trial Enrollment
}

\author{
Catriona Parker, BAppSc(MLS), MAppSc(MedSc)a ${ }^{a}$ Raymond Snyder, MBBS, MMed, FRACPb; \\ Michael Jefford, MBBS, MPH, MHlthServMt, PhD, FRACPc; David Dilts, PhD, MBA ${ }^{\text {; }}$; \\ Rory Wolfe, BSc, PhD ${ }^{\mathrm{e}}$; and Jeremy Millar, BMedSc, MBChB, FRANZCR, FACPMed ${ }^{\mathrm{a}, \mathrm{f}}$
}

\begin{abstract}
Background: A low proportion of adults with cancer are recruited to clinical trials. Cancer Council Victoria provides funding to clinical trial sites through its statewide Cancer Trials Management Scheme (CTMS). Historically, there appeared to be a relationship between budgetallocated funding and the number of patients recruited. A randomized controlled trial was conducted to test whether additional funding in 2013 would increase trial recruitment. Methods: A total of 18 trial centers ("sites") received usual CTMS funds, whereas 16 intervention sites received usual funds plus additional funds, proportional to recruitment in 2011; additional payments to sites in the intervention group ranged from $\$ 6,750$ to $\$ 234,000$ AUD $(\approx 6,750-\$ 234,000$ USD at the time). This represented an average $11.8 \%$ (interquartile range [IQR], $8.0 \%, 12.3 \%$ ) increase in sites' budgets. Sites were required to use the funds with the aim of increasing recruitment. The study end point was the number of new participants recruited to trials in 2013. An online survey assessed strategies used to increase recruitment. Results: The median number of new trial recruits per site in 2013 was 21 (IQR, 5-39) in the control arm and 12.5 (IQR, 3.5-44.5) in the intervention arm. The ratio of new trial recruitment numbers at the intervention sites compared with control sites in 2013, adjusting for respective 2012 numbers and institution type, was $0.99(95 \% \mathrm{Cl}, 0.69,1.43 ; P=.96)$. The survey revealed most intervention sites used funding to increase staffing. Conclusions: Additional funding at a site level did not lead to a contemporaneous increase in trial recruitment.
\end{abstract}

J Nat/ Compr Canc Netw 2017;15(9):1104-1110 doi: 10.6004/jnccn.2017.0150

\section{Background}

Clinical trials improve treatments for people affected by cancer and further our understanding of cancer biology; however, only a small percentage of adults with cancer participate in clinical trials. This is not a new phenomenon. In 1999, a systematic review identified factors

From the a Clinical Network, Cancer Council Victoria, bepartment of Oncology, St Vincent's Hospital, and 'Division of Cancer Medicine, Peter MacCallum Cancer Centre, Melbourne, Australia; dDivision of Management, Oregon Health and Science University, Portland, Oregon; and Department of Epidemiology and Preventive Medicine, Monash University, and ${ }^{\dagger}$ Alfred Health Radiation Oncology, The Alfred, Melbourne, Australia.

Submitted December 20, 2016; accepted for publication May 19, 2017. Dr. Snyder has disclosed that he has stock ownership in CSL Limited, and serves as a consultant for and is on an advisory board for Eli Lilly. Dr. Millar has disclosed that he owns stock in Resmed and Estia Health. The remaining authors have disclosed that they have no financial interests, arrangements, affiliations, or commercial interests with the manufacturers of any products discussed in this article or their competitors. limiting the quality, number, and progress of randomized controlled trials (RCTs). ${ }^{1}$ Recruitment was identified as one of these factors, and participation barriers were classified as clinician-related (eg, time constraints) and patient-related (eg, demands of the trial), all of which are still applicable today.

This study was funded by the Victorian Cancer Agency.

The abstract was presented at the 2015 ASCO Annual Meeting; May 29-June 2, 2015; Chicago, Illinois [J Clin Oncol 2015;33(Suppl 1):Abstract 6514], and Australia Clinical Trials Alliance 2015 International Clinical Trials Symposium; October 7-10, 2015; Sydney, Australia.

Author contributions: Study concept and design: Parker, Snyder, Jefford, Dilts, Millar. Data acquisition: Parker, Snyder. Data analysis and interpretation: Parker, Jefford, Dilts, Wolfe, Millar. Manuscript preparation: Parker, Snyder, Jefford, Dilts, Wolfe, Millar. Final approval of manuscript: Parker, Snyder, Jefford, Dilts, Wolfe, Millar.

Correspondence: Catriona Parker, BAppSc(MLS), MAppSc(MedSc), Clinical Network, Cancer Council Victoria, 615 St. Kilda Road, Melbourne, Victoria 3004, Australia. E-mail: catriona.parker@monash.edu 
Does Additional Funding Improve Trial Enrollment?

In Victoria, Australia's second most populous state, trial participation is also low. Cancer Council Victoria (CCV), a not-for-profit cancer charity, has financially supported clinical trials through its Cancer Trials Management Scheme (CTMS) since 1988. The CTMS collects annual data about patients enrolled into therapeutic cancer clinical trials and provides funding to sites (health service units or departments undertaking clinical trials) based on these numbers. Australia has a universal healthcare system and some privatized healthcare services; the Australian Government publically funds some sites and others are privately owned by for-profit entities.

When reviewing historical CTMS data, we observed a positive association between allocated CTMS funding and trial enrollment. We hypothesized that additional funding to clinical trial sites might increase trial enrollment and therefore undertook an RCT, the Additional Funding Intervention Trial (AFIT), to test this hypothesis. The trial also aimed to describe what strategies sites would use to increase accrual and to identify enrollment barriers from a site perspective.

\section{Methods}

This trial was approved by CCV's Institutional Research Review Committee.

\section{Overview of Trial and Existing CTMS}

The trial followed the same design as the existing CTMS so that the procedure was familiar and minimized any additional impost on site participants. The CTMS is undertaken annually; between February and March, sites submit their preceding calendar year's recruitment data to CCV for each active trial. Sites provide the number of new participants and the number of participants being followed up from previous years. A weighted capitation algorithm is then applied to allocate funding to each site. The AFIT study followed the same timelines, process, and collected data points. The usual CTMS funding continued during the trial to all sites. However for AFIT, only recruitment to interventional treatment trials of chemotherapy, radiotherapy, or surgical oncology modalities were included; supportive care trials were not included. The usual CTMS funding considers all trials that meet the WHO definition of a clinical trial. $^{2}$
Primary analysis compared the intervention and control sites' postintervention recruitment to eligible trials (2013 data), with adjustment for respective baseline recruitment to eligible trials (2012 data).

\section{Participating Sites}

There were 43 clinical trial sites funded by the CTMS in 2012; 5 sites were excluded from AFIT because they either had no recruitment for the preceding year (4 sites) or only recruited pediatric patients (1 site). Another 4 sites opted out, citing operational reasons. The remaining 34 sites underwent stratified block randomization based on their 2011 trial recruitment, which exhibited substantial variation across sites.

\section{Intervention}

Randomization was to 1 of 2 groups: an intervention group that received funding through the trial ("intervention funding") in addition to normal CTMS funding, or the control group, which received usual CTMS funding and a one-off incentive payment of $\$ 700$ AUD ( $\approx$ \$700 USD at the time) after completion of a survey. Intervention funding was intended to make a noticeable pro-rata difference to a site's budget, which was calculated based on previous recruitment.

No literature indicated how much funding was likely to be effective. The total amount of intervention funding was divided by the total number of new participants accrued across all intervention sites in 2011, which provided a payment amount per new participant ("dose") of $\$ 1,350$ AUD $(\approx \$ 1,350$ USD at the time). Each site was allocated intervention funding by multiplying the dose by the number of participants they reported from eligible trials in 2011. Additional payments to sites in the intervention group ranged from $\$ 6,750$ to $\$ 234,000$ AUD $(\approx \$ 6,750-\$ 234,000$ USD at the time) and represented a median increase of 300\% (interquartile range [IQR], 112.5\%, 459.0\%) relative to usual CTMS funding.

\section{Survey of Participating Sites}

All sites were surveyed in November 2013, toward the conclusion of the trial. Four online surveys were developed for clinical leads and trial managers at the intervention and control sites. The surveys asked multiple-response and open-ended questions, and were piloted using a sample of the intended study respon- 
dents (trial managers and clinical leads) and the trial steering committee members. The surveys were collected via Survey Monkey (www.surveymonkey.com).

Intervention sites were asked how they spent additional funds, their perception of the funding's effectiveness, and barriers to patient accrual at their institution. Sites allocated to the control group were asked the same questions in a hypothetical context. Surveys were emailed to staff at all randomized sites. Reminders were sent to individuals who had not responded within 2 weeks.

\section{Analysis Plan}

Limited funding was available over a defined period of time for this study (2 years). The trial captured almost all Victorian clinical trial sites available to $\mathrm{CCV}$, and we wanted to closely replicate the CTMS. Providing equal opportunity for all sites to obtain additional funding through randomization was an important consideration. With 34 sites, the study had $80 \%$ power to detect a $17 \%$ increase in recruitment (based on a simplified calculation from a Poisson model for recruitment per site, anticipating a mean 20 recruits per site in the control group and a 5\% type 1 error rate). Negative binomial regression was used to estimate the ratio of the number of new recruits in 2013 between the control and intervention groups, while adjusting for 2012 baseline data. A second analysis further adjusted for whether the site's trial department was in a public or private health service.

The model assumed that the logarithm (ln) of the recruitment count was linear in the explanatory factors and was of the form:

\section{$\ln ($ number of new recruits in 2013) $=b 0+b 1 *$ group + b2* $\ln ($ number of new recruits in 2012)}

The exponential function of $b 1\left(\mathrm{e}^{\mathrm{b} 1}\right)$ gives the ratio of the 2013 new recruitment in the intervention group compared with the 2013 new recruitment in the control group, allowing for sites' 2012 levels of new recruitment. To test for any differential effect of the intervention within the randomization strata, an interaction between group and strata was assessed in the negative binomial model. All analyses were performed using Stata, Version 12 (StataCorp LP, College Station, TX) and $P$ values $<0.05$ were considered significant.

\section{Results}

\section{Participating Sites}

Details of participating sites are shown in Table 1; 2 of the 3 largest sites were randomized to the control group. The distribution of public and private institutions was slightly imbalanced, with the control group having a higher percentage of public institutions.

\section{Recruitment Outcomes}

A summary of the recruitment outcomes and sitespecific changes in recruitment numbers from 2012 to 2013 are shown in Table 2. Allowing for 2012 new recruitment, the 2013 new recruitment rates in the intervention and control groups were estimated to be almost identical (ratio, 0.99; 95\% CI, 0.69-1.43), consistent with the null hypothesis of no difference between the groups $(P=.96)$ (Table 3). No evidence of a differential effect of intervention was seen across 2011 recruitment levels $\left(\chi_{3}^{2}=2.27 ; P=.5\right.$ for interaction). Table 3 also shows the results after adjustment for the type of institution (public vs private). This had little impact on the estimated intervention effect, as can be seen from the similar annual recruitment rate ratio (relative risk $[R R], 1.03 ; 95 \% \mathrm{CI}$, 0.72-1.45) upon adjustment to the original estimate of RR of 0.99 .

\section{Survey Data}

All trial managers responded to the survey; 16 control site clinical leads (89\%) and 15 intervention site clinical leads responded (94\%).

The intervention group commonly used the intervention funding to increase staffing, provide

\begin{tabular}{|lcc|}
\hline \multicolumn{2}{|c|}{ Table 1. Baseline Site Characteristics } \\
\hline Number of sites & 18 & Intervention \\
\hline $\begin{array}{l}\text { 2012 new recruits, } \\
\text { median (IQR) }\end{array}$ & $17.5(10.0,51.0)$ & 16 \\
\hline $\begin{array}{l}\text { Private/Public } \\
\text { Private }\end{array}$ & $2(11 \%)$ & $6(38 \%)$ \\
\hline Public & $16(89 \%)$ & $10(63 \%)$ \\
\hline Stratification group & \\
\hline$<15$ & $7(39 \%)$ & $7(44 \%)$ \\
\hline $15-34$ & $5(28 \%)$ & $4(25 \%)$ \\
\hline $35-99$ & $4(22 \%)$ & $4(25 \%)$ \\
\hline 100 & $2(11 \%)$ & $1(6 \%)$ \\
\hline
\end{tabular}

Abbreviation: IQR, interquartile range.

aBased on 2011 new recruitment numbers. 
Does Additional Funding Improve Trial Enrollment?

\begin{tabular}{|lcc|}
\hline $\begin{array}{l}\text { Table 2. Changes in Recruitment Numbers } \\
\text { (2012-2013) }\end{array}$ & $\begin{array}{c}\text { Control Arm, } \\
\text { Median (IQR) }\end{array}$ & $\begin{array}{c}\text { Intervention Arm, } \\
\text { Median (IQR) }\end{array}$ \\
\hline Outcome & $17.5(10.0,51.0)$ & $17.0(6.5,41.5)$ \\
\hline 2012 New recruits & $21.0(5.0,39.0)$ & $12.5(3.5,44.5)$ \\
\hline 2013 New recruits & $-2.5(-10.0,3.0)$ & $-2.0(-5.0,3.5)$ \\
\hline $\begin{array}{l}\text { Change in new } \\
\text { recruits }^{\mathrm{a}}\end{array}$ & & \\
\hline
\end{tabular}

Abbreviation: IQR, interquartile range.

a2013 new recruits minus 2012 new recruits.

staff training opportunities, improve infrastructure, open additional trials, or promote trials. The funding was also used to cover preexisting budget shortfalls (see Table 4). Control sites were asked what strategies they would use to increase accrual if they were granted substantial extra funding. Most managers and clinical leads agreed it would be used to increase staffing. Most sites reported that they were dependent on research grants for some or all of their employees' salaries. The most commonly cited barrier to recruitment to clinical trials for both intervention and control groups was inadequate experienced staffing levels. Trial-related barriers were also frequently mentioned, such as restrictive inclusion/ exclusion criteria, being unable to attract sponsors to open trials, unexpected early trial closure, or procedural delays.

Annual budgets of the sites varied enormously, with 4 sites having estimated annual budgets $<\$ 100,000$ AUD $(\approx \$ 100,000$ USD at the time $)$ and 5 sites with estimated annual budgets $>\$ 1$ million AUD $(\approx \$ 1$ million USD at the time). Additional funding to intervention sites was an average $11.8 \%$ (IQR, 8.0\%, 12.3\%) increase in their budgets. Onefifth of trial managers reported that their annual budget was adequate to undertake an effective trials program. Irrespective of the size of their current

\begin{tabular}{|c|c|c|}
\hline Model Outcome: Recruits 2013 & $\begin{array}{c}\text { Annual } \\
\text { Recruitment Rate } \\
\text { Ratio }(95 \% \mathrm{Cl})\end{array}$ & $P$ Value \\
\hline \multicolumn{3}{|l|}{ Adjusting for baseline a only } \\
\hline Group (intervention/control) & $0.99(0.69-1.43)$ & .96 \\
\hline \multicolumn{3}{|c|}{ Adjusting for baseline ${ }^{a}$ and institution } \\
\hline Group (intervention/control) & $1.03(0.72-1.45)$ & .89 \\
\hline Institution (public/private) & $1.51(0.95-2.38)$ & .08 \\
\hline
\end{tabular}

aBaseline $=2012$ recruitment numbers. budget, approximately one-third felt that 1.5 times the current budget was needed.

\section{Discussion}

Clinical trials are essential to translate laboratory discoveries to improved outcomes for patients. For example, there have been incremental improvements in the management of most childhood cancers, including acute lymphoblastic leukemia (ALL), with 5 -year survival now $>90 \% .^{3}$ More than half of all children with cancer, aged 0 to 10 years, participate in NCI-sponsored cooperative group trials. ${ }^{4}$ The reasons for improved outcomes in ALL are likely multifactorial; however, the contribution of clinical trial participation should not be underestimated.

Our trial aimed to determine whether additional funding to sites would lead to an increase in patient accrual. Although we found no evidence that this was the case, some contributing factors should be considered. In particular, a variety of factors that could not be controlled for or readily identified and/ or quantified during the trial may have influenced the outcome; for instance:

- Extra funding was being used for preexisting budget deficits.

- Trial duration was too short to allow sites time to employ additional experienced staff or observe any increase in accrual.

- There were no studies available that were suitable to open.

- Studies may have varied in complexity between 2012 and 2013, wherein more complex studies are likely to recruit fewer patients.

\section{Minimum Infrastructure and Money Management}

In undertaking our study, we assumed that sites were best positioned to know how to increase accrual and were therefore able to use the additional funding at their discretion for this purpose. We also assumed that the minimum infrastructure was present at every site to conduct trials efficiently and effectively. After our study ended, a peak body, the National Health and Medical Research Council, commenced work to define what constitutes a "Clinical Trials Ready" site, ${ }^{5}$ aiming to describe and articulate the minimum infrastructure for clinical trials. 
Parker et al

Table 4. How Additional Funding Was Used

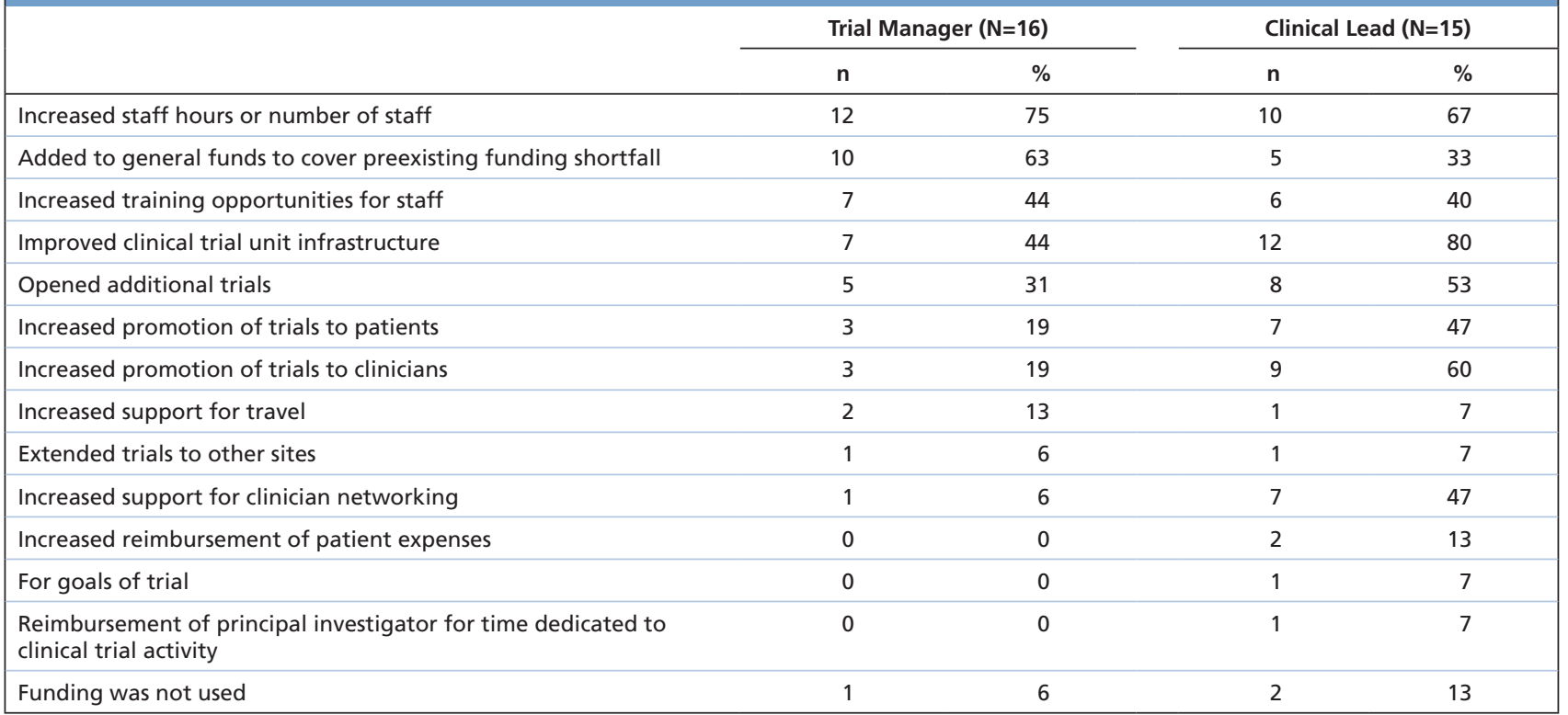

The survey of intervention sites revealed that, in some instances, the intervention funding was used to "catch-up." This could imply that minimum infrastructure was not present at the sites and/or there was a previous budget shortfall consideration. Commonly, budgets for cooperative groups, investigatorinitiated, and industry-sponsored trials are pooled. Seow et $\mathrm{al}^{6}$ described how sites rely on opening new studies to help cover the cost of follow-up in older studies. Perhaps, then, it is not surprising that sites reported the intervention funding was used to cover budget deficits that already existed.

Many respondents were dependent on research grants for some or all of their employees' salaries, and also reported inadequate staffing or high staff turnover. The inherent short-term nature of grants induces a cycle whereby it is difficult to attract and employ new staff, offer job security for current employees, and, thus, be able to take on new trials.

This study had a finite budget available and large variations in recruitment between sites. The methodology attempted to account for this by providing pro rata funds based on recruitment. However, if there is a threshold of effect in terms of the amount of money required to increase accrual, it may be higher than we could afford from our budget and was therefore unobserved.

\section{Trial Mix and Complexity}

Phase I trials inherently accrue fewer patients than large phase III trials. However, early-phase trials (including phase I /first-in-human studies) are generally far more complex. The mix of trials at each participating site was not controlled for; however, site randomization aimed to balance sites with similar profiles (including participation in early-stage trials).

Complex trial protocols are associated with an increased work burden. ${ }^{7,8}$ The use of intervention funding to increase staff and the identified barrier of maintaining staff levels could be direct results of increasingly complex trial protocols. Complex protocols are commonly associated with phase I studies; however, even phase II and some phase III protocols have an increased workload burden and accrue fewer patients compared with the previous paradigm of a relatively simple trial design. ${ }^{9}$ This is relevant for the present study given that the number of new recruits was the primary measure of the hypothesis. There are workload assessment tools using protocol acuity to help sites negotiate sponsor budgets and assess a protocol before committing to a trial. ${ }^{10-12}$ Such tools will be increasingly important for sites to manage trial/workload ratios given the mounting fiscal pressures on health services.

A confounding factor to consider is that individual clinical trial departments may not have increasing 
Does Additional Funding Improve Trial Enrollment?

patient accrual as a strategic objective; therefore, the goals of this study may not have aligned with individual site priorities and organizational reporting requirements. Alternatively, sites may have prioritized completing studies to which they had already committed. We did not attempt to capture this or whether the intervention sites recruited from higher acuity studies over the trial period. Future work might consider priorities from a site perspective and measuring protocol acuity when designing an intervention.

\section{Existing Strategies}

A Cochrane review examining strategies to improve recruitment to research studies found a considerable knowledge gap and concluded that "it is not possible to predict the effect most interventions will have on recruitment."13 The authors urged future researchers to ensure that evaluation of recruitment strategies is incorporated into studies. An updated Cochrane review in $2010^{14}$ echoed the earlier findings of Watson and Torgerson ${ }^{15}$ and found that trial design impacted patient accrual, but practical considerations limited widespread use of modified trial designs to enhance recruitment. None of our sites reported using trial design as a strategy to increase accrual, and we suggest this would be considered outside the influence of a typical site.

Our results suggest that increasing staffing may not lead to increased patient accrual to clinical trials. It would be valuable for future researchers to systematically assess other strategies thought to increase patient accrual to provide an evidence base for best practice conduct of clinical trials research.

\section{Trial Challenges}

Using patient recruitment as the only measurement in this study fitted into our established mechanism; however, increasing recruitment may not be the best end point in determining whether a funding intervention has been successful. Eligible patients may decline to participate for reasons related to protocol, inconvenience and lifestyle issues, financial considerations, or the pursuit of studies elsewhere. ${ }^{16}$ Trial protocols with lengthy inclusion and exclusion criteria are the new normal. It is possible that all eligible patients participated, but probably more likely that many more patients were screened than entered into the trial.
Similarly, some sites commented that there were no suitable trials available for them to open or that sponsors closed trials earlier than expected. It may be more pragmatic for future studies to use additional end points, such as the number of trials opened and recruiting to target; trials recruiting to target allow for statistically significant results and allow the sites to be viewed favorably by sponsors. Some sites reported using intervention funding to promote trials or open new trials. It is likely these strategies could not have been undertaken without additional staffing.

Our study participants believed that increasing staff levels is key to increasing patient accrual. This view was not supported by our findings, but we acknowledge a longer time frame of observation may have altered this finding.

\section{Limitations}

The duration of the study may have been too short to observe a positive effect on recruitment. In this study, recruitment in the intervention group actually decreased during the study period, possibly due to short-term fluctutations in recruitment. A longer period of evaluation might allow for year-on-year variations. We acknowledge that the unsustained nature of the interventional funding may have also posed a limitation and influenced how and on what individual sites spent their intervention funding. To overcome these challenges, we would recommend increasing the duration of the intervention funding and follow-up periods in future work. A further limitation is the lack of details regarding trial portfolio (including the complexity of studies) at each site.

\section{Conclusions}

We determined that increased funding at a site level did not translate to increased patient recruitment; however, this finding must be interpreted in the context of our methodological limitations. Increasing the study duration may have yielded different results, and we caution against applying these results more generally. Building on this initial study, we recommend a comprehensive exploration of site processes, systems, and organizational structures within which trials are conducted. Expanding staffing may not be a successful strategy to increase accrual. Future research should assess other strategies thought to increase accrual to clinical trials. 
Parker et al

\section{Acknowledgments}

The authors wish to acknowledge the following contributions: Ms. Kerry Haynes from the Centre for Behavioural Research in Cancer, Cancer Council Victoria, for her work with the survey component of this trial; and Ms.
Catherine Smith from the Department of Epidemiology and Preventive Medicine, Monash University, for her support in statistical analysis. Also, Dr. Jenni WilliamsSpence for her technical editing and proofreading of the manuscript.

\section{References}

1. Prescott RJ, Counsell CE, Gillespie WJ, et al. Factors that limit the quality, number and progress of randomized controlled trials. Health Technol Assess 1999;3:1-143.

2. Health Topics: Clinical Trials. World Health Organization Web site. Available at: http://www.who.int/topics/clinical_trials/en/. Accessed July $1,2012$.

3. Hunger SP, Lu X, Devidas M, et al. Improved survival for children and adolescents with acute lymphoblastic leukemia between 1990 and 2005: a report from the Children's Oncology Group. J Clin Oncol 2012;30:16631669.

4. Sateren WB, Trimble EL, Abrams J, et al. How sociodemographics, presence of oncology specialists, and hospital cancer programs affect accrual to cancer treatment trials. J Clin Oncol 2002;20:2109-2117.

5. Clinical Trials Ready - Concept and Criteria. Consultation Paper. Australian Government; National Health and Medical Research Council. April 2015. Available at: https://www.nhmrc.gov.au/_files_nhmrc/file/ research/clinical_trials/consulation_paper_clinical_trials_ready_initative. pdf. Accessed July 20, 2017.

6. Seow HY, Whelan P, Levine MN, et al. Funding oncology clinical trials: are cooperative group trials sustainable? J Clin Oncol 2012;30:1456-1461.

7. Getz KA, Wenger J, Campo RA, et al. Assessing the impact of protocol design changes on clinical trial performance. Am J Ther 2008;15:450-457.

8. Roche K, Paul N, Smuck B, et al. Factors affecting workload of cancer clinical trials: results of a multicenter study of the National Cancer Institute of Canada Clinical Trials Group. J Clin Oncol 2002;20:545-556.

9. Sjoquist KM, Zalcberg JR. Clinical trials-advancing national cancer care. Cancer Forum 2013;37:80-87.

10. Fowler DR, Thomas CJ. Protocol acuity scoring as a rational approach to clinical research management. Research Practitioner 2003;4:64-71.

11. Smuck B, Bettello $P$, Berghout $K$, et al. Ontario protocol assessment level: clinical trial complexity rating tool for workload planning in oncology clinical trials. J Oncol Pract 2011;7:80-84.

12. James $P$, Bebee $P$, Beekman $L$, et al. Creating an effort tracking tool to improve therapeutic cancer clinical trials workload management and budgeting. J Natl Compr Canc Netw 2011;9:1228-1233.

13. Mapstone J, Elbourne D, Roberts IG. Strategies to improve recruitment to research studies. Cochrane Database Syst Rev 2007:MR000013.

14. Treweek S, Mitchell E, Pitkethly M, et al. Strategies to improve recruitment to randomized controlled trials. Cochrane Database Syst Rev 2010:M000013.

15. Watson JM, Torgerson DJ. Increasing recruitment to randomised trials: a review of randomised controlled trials. BMC Med Res Methodol 2006;6:34.

16. Brintnall-Karabelas J, Sung $S$, Cadman ME, et al. Improving recruitmen in clinical trials: why eligible participants decline. J Empir Res Hum Res Ethics 2011;6:69-74.

Recorded Presentations from the
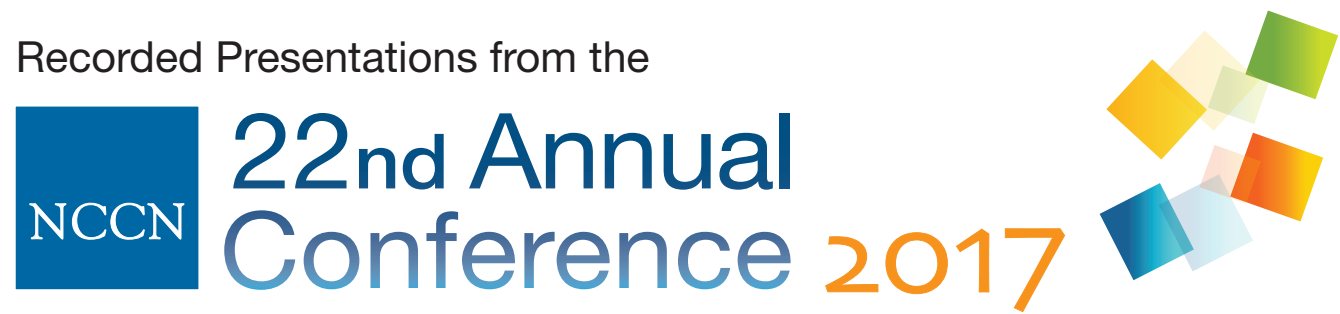

IMPROVING THE QUALITY, EFFECTIVENESS, AND EFFICIENCY OF CANCER CARE ${ }^{\text {TM }}$

Earn CME/CE credits online! More than 20 educational sessions featuring the latest advances in oncology care.

\section{education.nccn.org/ac2017}

This enduring material is approved for AMA PRA Category 1 Creditit for physicians and will award contact hours for nurses, pharmacists, and other health care professionals. Complete accreditation information is available online.

This activity is supported by educational grants from Ariad; Astellas and Medivation, Inc.; AstraZeneca; BTG International Inc.; Celgene Corporation; Celldex Therapeutics; Foundation Medicine; Genentech; Genomic Health, Inc.; Gilead Sciences, Medical Affairs; Lilly; Merrimack Pharmaceuticals, Inc.; Novartis; Pfizer; Prometheus Laboratories inc.; Taiho Oncology; and Takeda Oncology. This activity is supported by independent educational grants from

This educational activity is supported by a medical education grant from Exelixis, Inc. 